\title{
Palbociclib treatment alters nucleotide biosynthesis and glutamine dependency in A549 cells
}

Lindsey R. Conroy ${ }^{1,7}$, Pawel Lorkiewicz ${ }^{2,3}$, Liqing He ${ }^{3}$, Xinmin Yin ${ }^{3}$, Xiang Zhang ${ }^{3,6}$, Shesh N. Rai ${ }^{4,5,6}$

and Brian F. Clem ${ }^{1,6^{*}}$ (1)

\begin{abstract}
Background: Aberrant activity of cell cycle proteins is one of the key somatic events in non-small cell lung cancer (NSCLC) pathogenesis. In most NSCLC cases, the retinoblastoma protein tumor suppressor (RB) becomes inactivated via constitutive phosphorylation by cyclin dependent kinase (CDK) 4/6, leading to uncontrolled cell proliferation. Palbociclib, a small molecule inhibitor of CDK4/6, has shown anti-tumor activity in vitro and in vivo, with recent studies demonstrating a functional role for palbociclib in reprogramming cellular metabolism. While palbociclib has shown efficacy in preclinical models of NSCLC, the metabolic consequences of CDK4/6 inhibition in this context are largely unknown.
\end{abstract}

Methods: In our study, we used a combination of stable isotope resolved metabolomics using $\left[\mathrm{U}-{ }^{13} \mathrm{C}\right]$-glucose and multiple in vitro metabolic assays, to interrogate the metabolic perturbations induced by palbociclib in A549 lung adenocarcinoma cells. Specifically, we assessed changes in glycolytic activity, the pentose phosphate pathway (PPP), and glutamine utilization. We performed these studies following palbociclib treatment with simultaneous silencing of $R B 1$ to define the pRB-dependent changes in metabolism.

Results: Our studies revealed palbociclib does not affect glycolytic activity in A549 cells but decreases glucose metabolism through the PPP. This is in part via reducing activity of glucose 6-phosphate dehydrogenase, the rate limiting enzyme in the PPP. Additionally, palbociclib enhances glutaminolysis to maintain mitochondrial respiration and sensitizes A549 cells to the glutaminase inhibitor, CB-839. Notably, the effects of palbociclib on both the PPP and glutamine utilization occur in an RB-dependent manner.

Conclusions: Together, our data define the metabolic impact of palbociclib treatment in A549 cells and may support the targeting CDK4/6 inhibition in combination with glutaminase inhibitors in NSCLC patients with RB-proficient tumors.

Keywords: RB, Palbociclib, Metabolism, Glutaminolysis, PPP, Lung cancer

*Correspondence: brian.clem@louisville.edu

${ }^{1}$ Department of Biochemistry and Molecular Genetics, University of Louisville, Louisville, KY, USA

Full list of author information is available at the end of the article

\section{Background}

Lung cancer is among the most frequently diagnosed cancers and is the leading cause of cancer-related death worldwide, with an estimated 1.6 million deaths each year [1-3]. Of its subtypes, non-small cell lung cancer (NSCLC) accounts for $85 \%$ of all lung cancer diagnoses [2]. One of the hallmark events in NSCLC pathogenesis is

(c) The Author(s) 2020. This article is licensed under a Creative Commons Attribution 4.0 International License, which permits use, sharing, adaptation, distribution and reproduction in any medium or format, as long as you give appropriate credit to the original author(s) and the source, provide a link to the Creative Commons licence, and indicate if changes were made. The images or other third party material in this article are included in the article's Creative Commons licence, unless indicated otherwise in a credit line to the material. If material is not included in the article's Creative Commons licence and your intended use is not permitted by statutory regulation or exceeds the permitted use, you will need to obtain permission directly from the copyright holder. To view a copy of this licence, visit http://creativeco mmons.org/licenses/by/4.0/. The Creative Commons Public Domain Dedication waiver (http://creativecommons.org/publicdomain/ zero/1.0/) applies to the data made available in this article, unless otherwise stated in a credit line to the data. 
deregulation of the cell cycle $[4,5]$. Transition from G1 to $\mathrm{S}$ phase during cell cycle progression is tightly regulated by phosphorylation of the retinoblastoma protein tumor suppressor (RB) by the cyclin dependent kinase (CDK) 4/6-Cyclin D complex. Activity of the CDK4/6-Cyclin $\mathrm{D}$ complex is strongly inhibited by $\mathrm{p} 16$ which induces cell cycle arrest. In the majority of NSCLC cases, p16 is mutated, deleted, or epigenetically silenced, resulting in constitutive phosphorylation of RB and uncontrolled cell cycle progression $[4,5]$. Pharmacologically, CDK4/6 inhibition phenocopies p16 activity $[6,7]$.

Highly selective and potent small-molecule CDK4/6 inhibitors have shown anti-tumor activity both in vitro and in vivo [6, 7]. Among these inhibitors, palbociclib (PD-0332991) has been FDA-approved for the treatment of women with breast cancer [8]. Recently, palbociclib has shown efficacy either alone or as combination therapy in preclinical studies of NSCLC [9-13]. Mechanistically, the functional consequences of CDK4/6 inhibition apart from cell cycle arrest in NSCLC are largely unknown; yet, metabolic reprogramming in response to palbociclib has revealed unique targetable vulnerabilities in several cancers including pancreatic, colorectal, and leukemia [14-16]. As such, we aimed to characterize the metabolic phenotype induced by palbociclib in NSCLC.

Herein, we report that palbociclib treatment decreases nucleotide biosynthesis and increases glutamine utilization without altering glycolysis in A549 lung adenocarcinoma cells. Specifically, palbociclib-induced glutamine dependency sensitizes A549 cells to glutaminase inhibition. Together, these data expand our knowledge of understanding of the metabolic consequences of CDK4/6 inhibition in NSCLC.

\section{Methods}

\section{Cell culture}

The A549 lung adenocarcinoma cells were obtained from ATCC and cultured in DMEM (Gibco, Cat. No. 11965126) supplemented with $10 \%$ fetal bovine serum (Gibco, Cat. No. 10438034) and $50 \mu \mathrm{g} / \mathrm{mL}$ gentamicin sulfate (Gibco, Cat. No. 15750-078). Cells were maintained at $5 \% \mathrm{CO}_{2}$ at $37{ }^{\circ} \mathrm{C}$. Palbociclib (PD-0332991) (Cat. No. S1116) and CB-839 (Cat. No. S7655) were purchased from Selleck Chemicals.

\section{Cell transfections}

Using Lipofectamine RNAiMAX reagent (Invitrogen, Cat. No. 13778150), A549 cells were transiently transfected with control siRNA (Ambion, Silencer Select Negative Control \#2, Cat. No. 4390826) or siRNA targeted to RB1 (Ambion, Silencer Select s523, Cat. No. 4390824) for $24 \mathrm{~h}$ prior to palbociclib treatment.

\section{Cell proliferation and viability}

A549 cells were seeded at 120,000 in 6-well plates (Corning, Cat. No. 3516), followed by transfection and treatment with $1 \mu \mathrm{M}$ palbociclib or vehicle control. For glutaminase inhibition studies, cells were seeded at 25,000 in 24-well plates (Corning, Cat. No. 3526), followed by 25 nM CB-839 treatment. Forty-eight hours post-treatment, cell number was determined by Trypan Blue (Gibco, Cat. No. 15250-061) exclusion and enumeration using a hemocytometer.

\section{Glucose uptake}

A549 cells were seeded at 120,000 in 6-well plates (Corning, Cat. No. 3516). Cells were transfected and treated with $1 \mu \mathrm{M}$ palbociclib or vehicle control for $48 \mathrm{~h}$ prior to performing the assay. Cells were then incubated in glucose-free DMEM (Gibco, Cat. No. 11966-025) for $30 \mathrm{~min}$, followed by incubation with $5 \mu \mathrm{L}$ of $2-\left[{ }^{14} \mathrm{C}\right]$-deoxyglucose $(0.25 \mu \mathrm{Ci} / \mathrm{mL}$; Perkin Elmer, Cat. No. NEC720A050UC $)$ and three subsequent washes with ice-cold glucose-free DMEM. Lysates were collected in $500 \mu \mathrm{L}$ of $0.1 \%$ SDS and scintillation counts (counts/min) were measured using $350 \mu \mathrm{L}$ of cell lysate on a Tri-Carb 2910 liquid scintillation analyzer (Perkin Elmer Life Sciences). Protein concentration was determined using the BCA assay (Pierce, Cat. No. 23225) according to the manufacturer's protocol. Counts were normalized to $\mu \mathrm{g}$ of protein.

\section{Glycolysis stress test}

A549 cells were seeded at 120,000 in 6-well plates (Corning, Cat. No. 3516). Cells were transfected and treated with $1 \mu \mathrm{M}$ palbociclib or vehicle control for 48 or $120 \mathrm{~h}$ prior to performing the assay. ECAR measurements were conducted using a Seahorse XFe96 analyzer according to manufacturer's protocol. One day prior to performing the assay, cells were reseeded at 35,000 in XFe96 cell culture plates and incubated $5 \% \mathrm{CO}_{2}$ at $37^{\circ} \mathrm{C}$. One hour prior to analysis, growth medium was replaced with assay medium (DMEM minus phenol red and sodium bicarbonate (Corning, Cat. No. 90-013-PB) that is supplemented with $2 \mathrm{mM}$ L-glutamine, $\mathrm{pH}$ 7.4) and incubated in a non- $\mathrm{CO}_{2}$ incubator. During the assay, $10 \mathrm{mM}$ glucose (Sigma, Cat. No. G8270), $1 \mu \mathrm{M}$ oligomycin (Sigma, Cat. No. 495455), and $50 \mathrm{mM}$ 2-deoxyglucose (Sigma, Cat. No. D8375) were sequentially injected into each well in accordance with standard protocols. Absolute rates $(\mathrm{mpH} / \mathrm{min})$ were normalized to $\mu \mathrm{g}$ of protein.

\section{Mito stress test}

A549 cells were seeded at 120,000 in 6-well plates (Corning, Cat. No. 3516). Cells were transfected and treated with $1 \mu \mathrm{M}$ palbociclib or vehicle control for $48 \mathrm{~h}$ prior 
to performing the assay. OCR measurements were conducted using a Seahorse XFe96 analyzer according to manufacturer's protocol. One day prior to performing the assay, cells were reseeded at 35,000 in XFe96 cell culture plates and incubated in $5 \% \mathrm{CO}_{2}$ at $37{ }^{\circ} \mathrm{C}$. One hour prior to analysis, growth medium was replaced with assay medium (DMEM minus phenol red and sodium bicarbonate (Corning, Cat. No. 90-013-PB) that is supplemented with $1 \mathrm{mM}$ pyruvate, $2 \mathrm{mM} \mathrm{L}$-glutamine, and $10 \mathrm{mM}$ glucose, $\mathrm{pH}$ 7.4) and incubated in a non- $\mathrm{CO}_{2}$ incubator. During assay, $1 \mu \mathrm{M}$ oligomycin (Sigma, Cat. No. 495455), $2 \mu \mathrm{M}$ FCCP (Sigma, Cat. No. C2920), and $1 \mu \mathrm{M}$ rotenone/antimycin A (Sigma Cat. No. R8875 and A8674) were sequentially injected into each well in accordance with standard protocols. Absolute rates ( $\mathrm{p} \mathrm{moles} / \mathrm{min}$ ) were normalized to $\mu \mathrm{g}$ of protein.

\section{Mito fuel flexibility assay}

A549 cells were seeded at 120,000 in 6-well plates (Corning, Cat. No. 3516). Cells were transfected and treated with $1 \mu \mathrm{M}$ palbociclib or vehicle control for $48 \mathrm{~h}$ prior to performing the assay. OCR measurements were conducted using a Seahorse XFe96 analyzer according to manufacturer's protocol. One day prior to performing the assay, cells were reseeded at 35,000 in XFe96 cell culture plates and incubated $5 \% \mathrm{CO}_{2}$ at $37{ }^{\circ} \mathrm{C}$. One hour prior to analysis, growth medium was replaced with assay medium (DMEM minus phenol red and sodium bicarbonate (Corning, Cat. No. 90-013-PB) that is supplemented with $1 \mathrm{mM}$ pyruvate, $2 \mathrm{mM} \mathrm{L}$-glutamine, and $10 \mathrm{mM}$ glucose, $\mathrm{pH}$ 7.4) and incubated in a non- $\mathrm{CO}_{2}$ incubator. Capacity and dependency for fatty acid, glutamine, and glucose oxidation were determined through injections of the following inhibitors in accordance with standard protocols: $4 \mu \mathrm{M}$ etomoxir (Sigma, Cat. No. E1905) (inhibits long chain fatty acid oxidation), $3 \mu \mathrm{M}$ BPTES (Sigma, Cat. No. SML0601) (inhibits glutamine oxidation), and $2 \mu$ M UK5099 (Sigma, Cat. No. PZ0160) (inhibits glucose oxidation). The percent capacity for each fuel source was determined by dividing the observed OCR under inhibition of the two other pathways by the OCR observed when all pathways are inhibited. The percent dependency for each fuel source was determined by dividing the OCR observed when that specific pathway is inhibited by the OCR observed when all pathways are inhibited. Absolute rates ( $\mathrm{p}$ moles $/ \mathrm{min}$ ) were normalized to $\mu \mathrm{g}$ of protein.

\section{Glucose 6-phosphate dehydrogenase activity assay}

A549 cells were seeded at 120,000 in 6-well plates (Corning, Cat. No. 3516). Cells were transfected and treated with $1 \mu \mathrm{M}$ palbociclib or vehicle control for $48 \mathrm{~h}$ prior to performing the assay. The activity of G6PD from cell lysates was measured following the manufacturer's protocol using the Glucose-6-Phosphate Dehydrogenase Activity Assay Kit (Sigma, Cat. No. MAK015-1KT) and normalized to $\mu \mathrm{g}$ of protein.

\section{$\left[\mathrm{U}-{ }^{13} \mathrm{C}\right]$-glucose tracer studies}

Cells were seeded at $1 \times 10^{6}$ cells in $10 \mathrm{~cm}$ dishes (Corning, Cat. No. 430167) and treated with palbociclib or vehicle control for $48 \mathrm{~h}$. Cells were then labeled for $6 \mathrm{~h}$ with DMEM supplemented with $1 \mathrm{~g} / \mathrm{L}$ $\left[\mathrm{U}-{ }^{13} \mathrm{C}\right]$-glucose (Sigma, Cat. No. 38974 ) and $10 \%$ dialyzed fetal bovine serum (Gibco, Cat. No. A3382001). Cells were washed three times in ice-cold $1 \times$ PBS and quenched with acetonitrile. Metabolites were extracted in acetonitrile:water:chloroform $(2 \mathrm{~mL}: 1 \mathrm{~mL}: 740 \mu \mathrm{L})$. Samples were centrifuged at $3000 \times g$ for $20 \mathrm{~min}$ at $4{ }^{\circ} \mathrm{C}$ to separate the polar, lipid, and cell debris layers. The remaining cell debris was re-extracted with $500 \mu \mathrm{L}$ chloroform:methanol:butylated hydroxytoluene $(2: 1: 1 \mathrm{mM})$ and centrifuged at $22,000 \times g$ for $20 \mathrm{~min}$ at $4{ }^{\circ} \mathrm{C}$. The residual polar and lipid fractions were combined with their respective fractions from the first extraction. The polar fraction was vacuum-dried by lyophilization. The dried sample was dissolved in $100 \mu \mathrm{L}$ $50 \%$ acetonitrile and vigorously vortex-mixed for $3 \mathrm{~min}$. After centrifugation at $14,000 \mathrm{rpm}$ and $4{ }^{\circ} \mathrm{C}$ for $20 \mathrm{~min}$, $80 \mu \mathrm{L}$ of supernatant was collected for 2DLC-MS/MS analysis.

\section{DLC-MS/MS analysis and data processing}

All samples were analyzed in a random order on a Thermo Q Exactive HF Hybrid Quadrupole-Orbitrap Mass Spectrometer coupled with a Thermo DIONEX UltiMate 3000 HPLC system (Thermo Fisher Scientific, Waltham, MA, USA). The UltiMate 3000 HPLC system was equipped with a reverse phase chromatography (RPC) column and a hydrophilic interaction chromatography (HILIC) column that were configured in parallel to form a parallel 2DLC-MS system [17]. To obtain full MS data, every sample was analyzed by the parallel 2DLC-MS in positive mode $(+)$ and negative mode $(-)$, respectively. For metabolite identification, one unlabeled sample in each sample group was analyzed by 2DLCMS/MS in positive mode $(+)$ and negative mode $(-)$ to acquire MS/MS spectra at three collision energies (20, 40 and $60 \mathrm{eV})$.

\section{Data analysis for 2DLC-MS/MS}

Full MS.raw files were first converted to.mzML format with msConvert tool, a part of an open-source ProteoWizard suite, described in detail by Adusumilli and Mallick [18]. Isotopologue peak deconvolution and assignments were performed using El-MAVEN (Elucidata) [19]. Peaks were assigned using a metabolite list 
generated and verified using full scan MS and MS/MS spectra of unlabeled samples, as described previously [20-22]. The metabolite list contained metabolite names and corresponding molecular formulae used to generate theoretical $\mathrm{m} / \mathrm{z}$ values for all possible isotopologues, and retention times for each metabolite. El-MAVEN parameters for compound library matching were as follows: EIC Extraction Window $\pm 7 \mathrm{ppm}$; Match Retention Time $\pm 0.60 \mathrm{~min}$. For ${ }^{13} \mathrm{C}$ isotopologue peak detection, the software criteria were set as follows: Minimum Isotope-parent correlation 0.20; Isotope is within 5 scans of parent; Abundance threshold 1.0; Maximum Error To Natural Abundance 100\%. All assignments were visually inspected and compared to unlabeled samples for reference. The peak list with corresponding abundances was exported to a comma-separated (CSV) file and uploaded to the Polly workflow (Elucidata) for natural abundance correction and calculation of total pool size for each metabolite (by summing peak areas of each detected isotopologue) using Polly Isocorrect module. Finally, the data were downloaded and plotted using Microsoft Excel and GraphPad Prism software.

\section{Real time-PCR}

Total RNA was isolated from cell pellets using the RNeasy Mini Kit (Qiagen, Cat. No. 74104) according to the manufacturer's protocol. The resulting total RNA $(1 \mu \mathrm{g})$ was converted to cDNA using the High-Capacity RNA-to cDNA kit (Applied Biosystems, Cat. No. 4387406). Gene expression was determined by qPCR using the following Taqman Gene Expression Assays: RB1 (Hs01078066_ $\mathrm{m} 1$ ), RRM1 (Hs01040698_m1), RRM2 (Hs00357247_ g1), PRPS2 (Hs00267624_m1), G6PD (Hs0166169_m1), ASCT2 (Hs01056542_m1), GLS (Hs01014020_m1), and GLUD1 (Hs01632647_g1). $\beta$-actin (Hs01060665_g1) was used as an internal control. Data are reported as the log (base 2) of the fold change with respect to siNeg treated with vehicle control.

\section{Immunoblotting}

Whole-cell lysates were prepared in RIPA lysis buffer (Pierce, Cat. No. 89900) containing protease and phosphatase inhibitors. $20 \mu \mathrm{g}$ of protein was separated on $10 \%$ Bolt Bis-Tris gels (Invitrogen, Cat. No. nw00102box) and transferred to PVDF membrane. Membranes were blocked with 5\% BSA (Fisher Bioreagents, Cat. No. BP1600-100) in TBS-T and probed with 1:1000 dilution of anti-phospho-Rb (Cell Signaling, \#3590S) overnight at $4{ }^{\circ} \mathrm{C}$. Membranes were washed and incubated with HRP-conjugated anti-rabbit (1:5000) secondary antibodies (Invitrogen, Cat. No. 32260) for $1 \mathrm{~h}$. Protein detection was performed by ECL chemiluminescent reagent (GE Healthcare Amersham, Cat. No. RPN2232) exposure.
1:20,000 dilution of anti- $\beta$-actin antibody (Sigma, A2228) was used to assess protein loading.

\section{Statistical analysis}

Statistical analyses were carried out using GraphPad Prism. As described in their respective figure legends, analyses were performed using student's t-test and twoway ANOVA with Sidak's post hoc multiple comparisons. Within the two-factor ANOVA, interaction term was primarily included in the analysis and was dropped if found not to be significant. Statistical significances between any two groups are as follows: " $\mathrm{p}<0.05,{ }^{* *} \mathrm{p}<0.01$, and **:" $\mathrm{p}<0.001$.

\section{Results}

Palbociclib treatment does not alter glycolysis in A549 cells To assess the role of CDK4/6 inhibition on metabolism in NSCLC, we utilized A549 lung adenocarcinoma cells. While these cells express wild-type RB, they harbor a p $16^{\mathrm{INK} 4 \mathrm{~A}}$ deletion that results in constitutive RB hyperphosphorylation and inactivation. Treatment with palbociclib inhibits the phosphorylation of RB and results in decreased cell proliferation (Additional file 1: Figure S1A, B). Recent studies have demonstrated that palbociclib alters glucose metabolism in multiple cancer subtypes [23-25]. To monitor the effect of palbociclib on glucose uptake, we performed radiolabeled glucose uptake assay using 2- $\left[{ }^{14} \mathrm{C}\right]$-deoxyglucose in A549 cells. As CDK4/6 inhibition can have metabolic effects independent of RB, these studies were performed following palbociclib treatment with simultaneous knockdown of $R B 1$ (Additional file 1: Figure S2). We observed no significant change in glucose uptake upon palbociclib treatment in A549 cells (Fig. 1a). To assess glycolytic function in response to palbociclib treatment, we performed a glycolysis stress test using the Seahorse XFe96 analyzer. Similar to glucose uptake, we observed no difference in the extracellular acidification rate (ECAR) upon palbociclib treatment (Fig. 1b-f), suggesting palbociclib does not alter glycolysis in A549 cells.

To define global changes in glucose utilization resulting from palbociclib treatment, we performed $\left[\mathrm{U}^{13} \mathrm{C}\right]$ glucose tracer studies. Utilization of ubiquitously labeled glucose results in the intracellular generation of ${ }^{13} \mathrm{C}$ labeling of the hexose and triose sugar intermediates within the glycolytic pathway; leading to fully labeled pyruvate $(\mathrm{m}+3)$ and lactate $(\mathrm{m}+3)$ (Fig. 2a) Palbociclib has been shown to increase glycolytic flux in both breast and pancreatic cancers [14, 24, 25]; however, consistent with our glycolysis stress test findings, palbociclib did not alter glucose carbon incorporation into glycolytic intermediates in A549 cells (Fig. 2b-g). Our data suggest that 

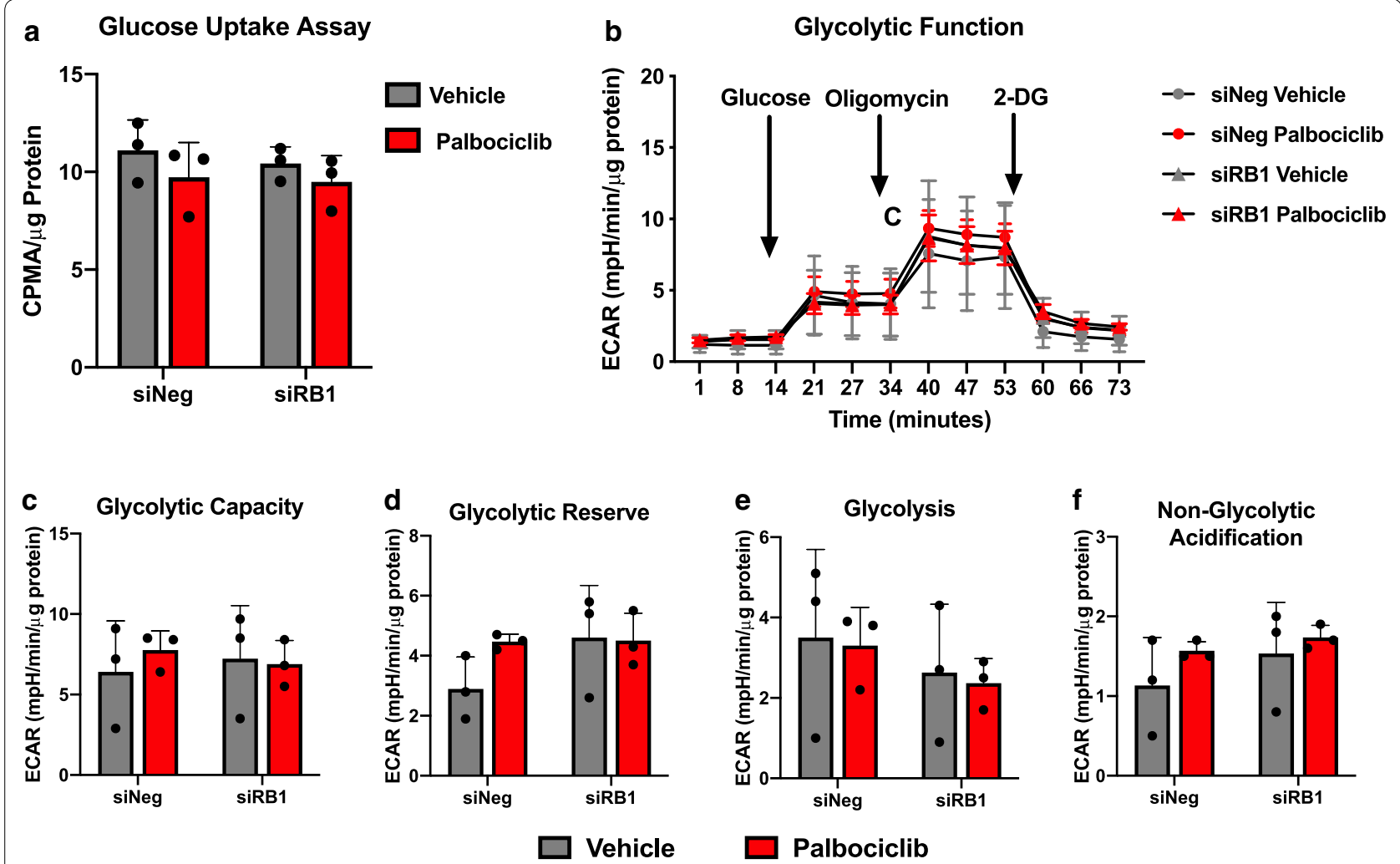

Fig. 1 Palbociclib does not alter glucose uptake or glycolytic function in A549 cells. a Glucose uptake assessed by intracellular 2-[ $\left.{ }^{14} \mathrm{C}\right]-$ deoxyglucose in A549 cells following knockdown of RB and 48-h treatment with $1 \mu \mathrm{M}$ palbociclib. b Glycolysis stress test measured in A549 cells following knockdown of RB and 48-h treatment with $1 \mu \mathrm{M}$ palbociclib using a Seahorse XFe96 Analyzer. c-f ECAR assessment of c glycolytic capacity, d glycolytic reserve, e glycolysis, and $\mathbf{f}$ non-glycolytic acidification. Values represent mean \pm SD, analyzed by two-way ANOVA with Sidak's post hoc multiple comparisons ( $n=3$, independent experiments)

the palbociclib-mediated effects on glycolysis may be tissue-specific.

\section{CDK4/6 inhibition decreases glucose flux through the PPP}

We then examined whether glucose metabolism within tangential pathways, such as the pentose phosphate pathway, may be altered by palbociclib treatment. Metabolism of fully labeled ${ }^{13} \mathrm{C}$-glucose generates different metabolite labeling patterns within PPP intermediates, including the 5 -carbon labeled ribose moiety $(m+5)$ on de novo synthesized nucleotides from ribose-5-phosphate as well as 7 labeled carbon sedoheptulose-7-phosphate $(m+7)$. We observed decreased $m+5$ isotopologue labeling of several nucleotides (Fig. 3a) as well as diminished glucose carbon incorporation into sedoheptulose 7-phosphate (Fig. 3b). Together, these data suggest palbociclib decreases glucose metabolism through the PPP. Consistent with our labeling data, palbociclib decreased glucose 6-phosphate dehydrogenase (G6PD) activity in an RB-dependent manner (Fig. 3c). Additionally, we measured expression of G6PD and other genes within nucleotide synthesis including phosphoribosyl pyrophosphate synthetase 2 (PRPS2) and ribonucleotide reductase $1 / 2$ (RRM1/2). Palbociclib decreased expression of $R R M 2$ in an RB-dependent manner but does not significantly alter expression of these other genes within nucleotide synthesis (Fig. 3d-g).

Palbociclib increases oxygen consumption in A549 cells CDK4/6 inhibition has been reported to increase both mitochondrial mass and oxygen consumption in pancreatic cancer [14]. As such, we assessed mitochondrial activity by measurement of the oxygen consumption rate (OCR) in A549 cells following palbociclib treatment. Consistent with other cancer types [14, 15], we observed a significant increase in several parameters of mitochondrial activity including basal $(\mathrm{p}=0.002)$ and non-mitochondrial respiration $(\mathrm{p}=0.01)$ and ATP production $(\mathrm{p}=0.034)$ (Fig. 4). While two-way ANOVA revealed no interaction between palbociclib treatment and RB status $(\mathrm{p}=0.113)$, palbociclib increased basal OCR 1.4-fold, which was reduced to 1.2-fold in the absence of RB.

To determine if the increase in basal oxygen consumption upon palbociclib treatment is the result of 

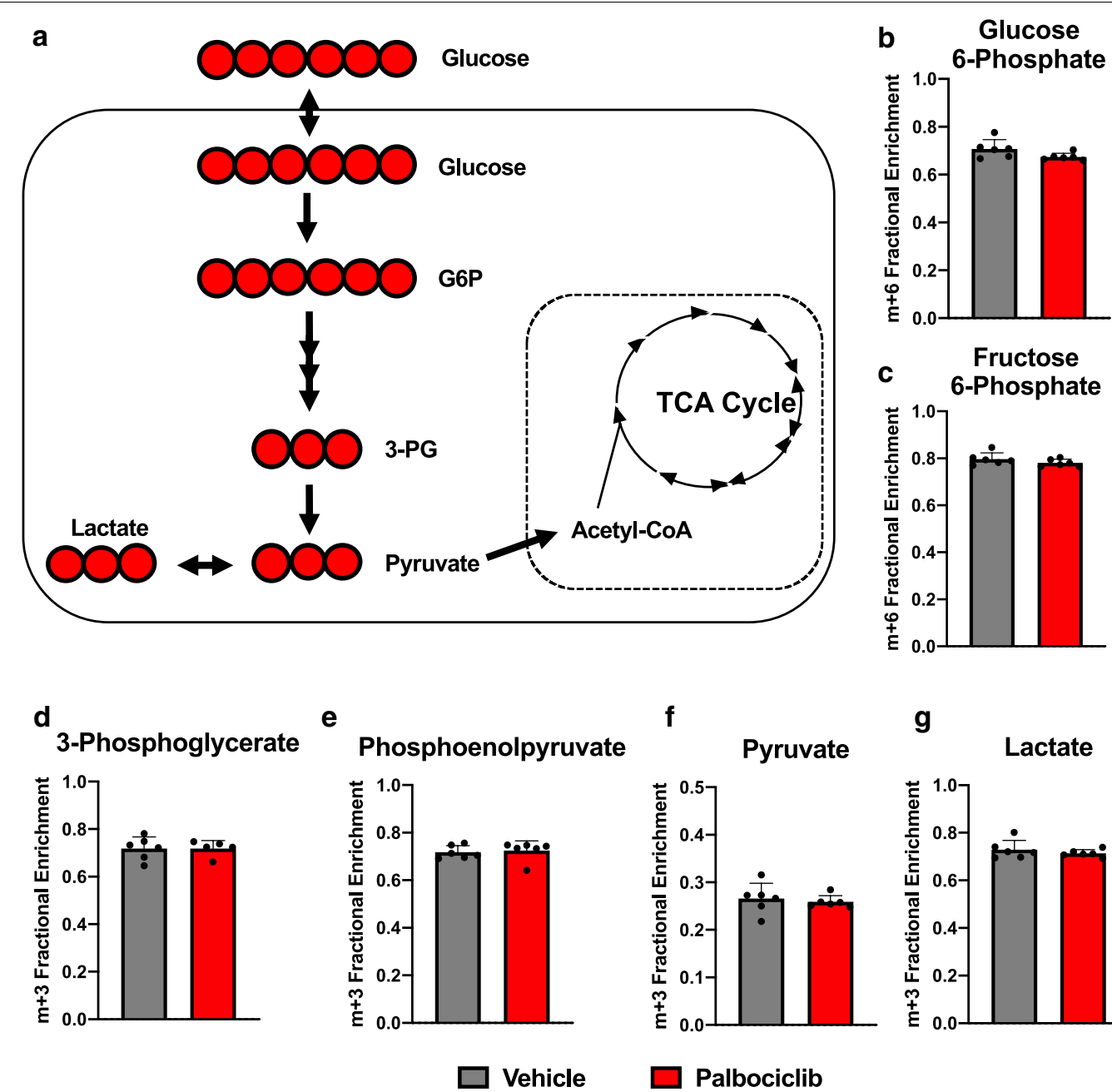

\section{f}

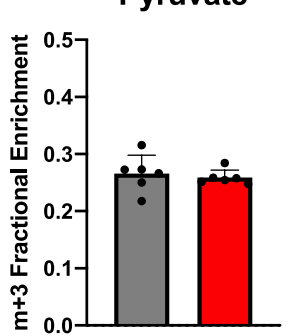

g

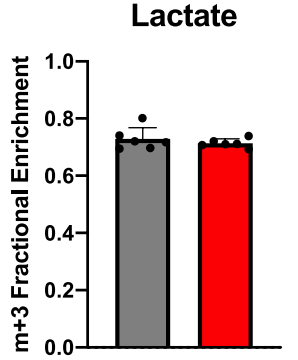

\section{Palbociclib}

Fig. 2 Palbociclib does not alter glucose carbon incorporation into glycolytic intermediates in A549 cells. a Cartoon of $\left[\mathrm{U}-{ }^{13} \mathrm{C}\right]$-glucose fate mapping though glycolysis. Red circles indicate ${ }^{13} \mathrm{C}$. Fractional enrichment of $\mathrm{m}+6$ labeled $\mathbf{b}$ glucose 6 -phosphate and $\mathbf{c}$ fructose 6 -phosphate, and $\mathrm{m}+3$ labeled $\mathbf{d}$ 3-phosphoglycerate, e phosphoenolpyruvate, $\mathbf{f}$ pyruvate, and $\mathbf{g}$ lactate following 48-h treatment with $1 \mu \mathrm{M}$ palbociclib. Values represent mean $\pm S D$, analyzed by unpaired student's t-test $(n=6$, technical replicates)

increased glucose oxidation, we measured TCA cycle metabolites following $\left[\mathrm{U}_{-}{ }^{13} \mathrm{C}\right]$-glucose labeling in A549 cells. Pyruvate $(m+3)$ generated from glycolysis can be metabolized to lactate by lactate dehydrogenase (LDH) or oxidized in the TCA cycle through pyruvate dehydrogenase $(\mathrm{PDH})$ or pyruvate carboxylase (PC). $\mathrm{PDH}$ entry of pyruvate carbon is indicated by $\mathrm{m}+2$ (1st turn) $/ \mathrm{m}+4$ (2nd turn) isotopologues, while PC activity is observed by $m+3$ isotopologue labeling of TCA intermediates (Fig. 5a). While there was no consistent effect on PDH-mediated pyruvate carbon entry $(m+2$ or $\mathrm{m}+4)$, we found that palbociclib increased $\mathrm{m}+3$ labeling of several TCA metabolites, suggesting palbociclib may increase PC activity for anaplerosis in A549 cells (Fig. 5b-e).
Palbociclib sensitizes A549 cells to glutaminase inhibition in an RB-dependent manner

We next sought to determine whether palbociclib alters the capacity to metabolize or the dependency on specific TCA fuels such as glucose, glutamine, or fatty acids for mitochondrial function using the Mito Fuel Flex assay on the Seahorse XFe96 analyzer. Palbociclib had no effect on the overall capacity of A549 cells to utilize glucose but did decrease the cells' dependency on glucose to maintain basal respiration, indicating glucose is not the sole fuel source for the TCA cycle in palbociclib treated cells (Fig. 6a, d). While palbociclib increases the capacity to oxidize fatty acids, it has no effect on the cells' dependency for fatty acid oxidation, suggesting that inhibition of fatty acid oxidation would have no effect on the 

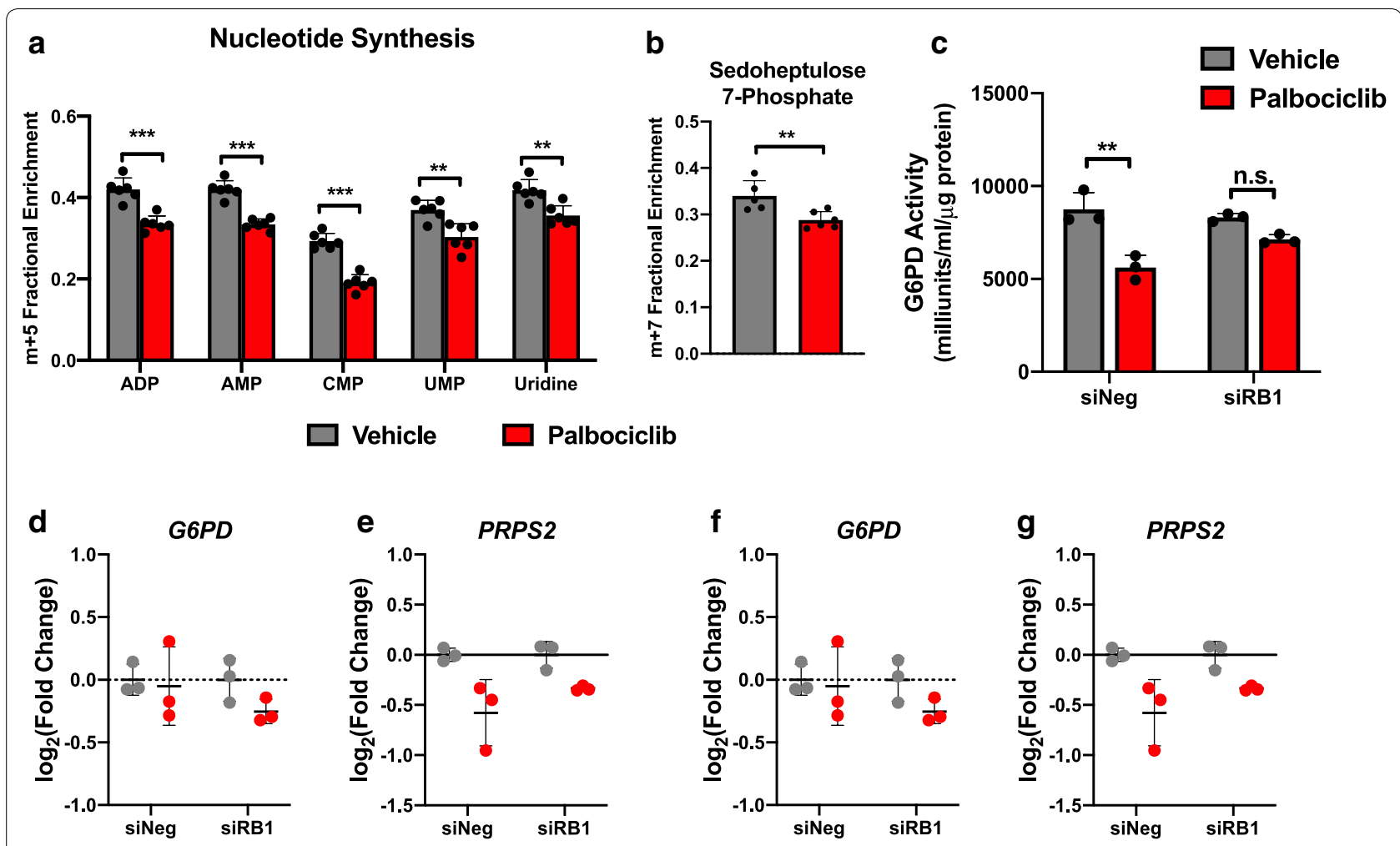

\section{- Vehicle Palbociclib}

Fig. 3 Palbociclib decreases glucose carbon incorporation into PPP intermediates, in part, via decreased G6PD activity. a Fractional enrichment of $m+5$ labeled nucleotides and nucleosides, and $\mathbf{b} m+7$ sedoheptulose 7-phosphate following 48-h treatment with $1 \mu$ M palbociclib. Values represent mean $\pm S D$, analyzed by unpaired student's t-test $(n=6$, technical replicates). Statistical significances between each group are as follows: ${ }^{* *} p<0.01$ and ${ }^{* * *} p<0.001$. c G6PD activity assessed in A549 cells following knockdown of RB and 48-h treatment with $1 \mu M$ palbociclib. $\mathbf{d}-\mathbf{g}$ qPCR analysis of glucose 6-phosphate dehydrogenase (G6PD), phosphoribosyl pyrophosphate synthetase 2 (PRPS2), and ribonucleotide reductase $1 / 2$ (RRM1/2) in A549 cells following knockdown of RB and 48-h treatment with $1 \mu \mathrm{M}$ palbociclib. c-g Values represent mean \pm SD, analyzed by two-way ANOVA with Sidak's post hoc multiple comparisons ( $n=3$, independent experiments). Statistical significances between each group are as follows: ${ }^{* *} \mathrm{p}<0.01$

viability of palbociclib treated cells (Fig. 6b, e). Notably, we observed an increase in both glutamine capacity and dependency in A549 cells upon palbociclib treatment (Fig. 6c, f).

The enzyme glutaminase converts glutamine to glutamate and is highly expressed in cancer cells [26-28]. CB-839 is a highly potent glutaminase inhibitor that has shown anti-tumor activity in both in vitro and in vivo models of lung cancer [29]. We hypothesized that the observed increase in glutamine dependency may sensitize palbociclib treated cells to glutaminase inhibition. Although palbociclib does not significantly alter glutaminase (GLS) gene expression (Fig. 6g), we observed a significant proliferative decrease in cells treated in combination with the glutaminase inhibitor CB-839 and palbociclib compared with palbociclib or CB-839 alone (Fig. 6h). Additionally, statistical analysis found a significant interaction between inhibitor sensitivity and RB status ( $\mathrm{p}<0.0001)$, suggesting $R B$ expression is necessary for palbociclib to exert its effect on glutamine dependency. Together, our data highlight that palbociclibinduced metabolic adaptations have the potential to be therapeutically exploited.

\section{Discussion}

While targeting cancer metabolism for therapeutic intervention has been extensively studied [30-34], there is a growing appreciation for cell cycle proteins that display metabolic regulatory functions [6, 35-38]. Additionally, with the increase in utilization of CDK4/6 inhibitors in the clinic, there is a need for better understanding of the metabolic consequences of CDK4/6 inhibition. In the current study, we report that activation of RB via treatment with the CDK4/6 inhibitor palbociclib in A549 lung adenocarcinoma cells results in a metabolic shift wherein palbociclib alters aspects of 
a

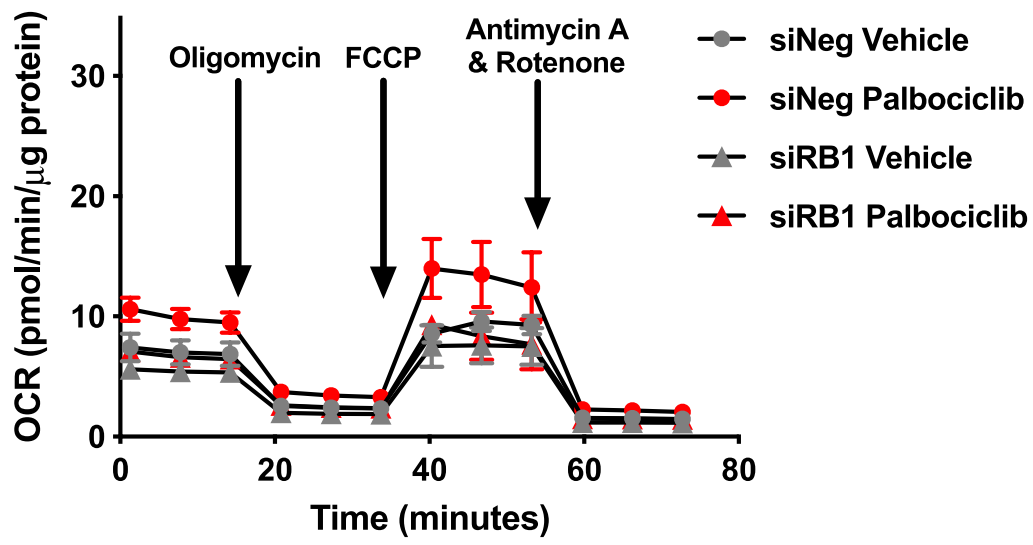

b

Basal Respiration

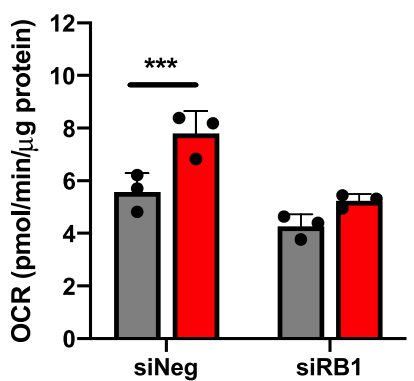

-

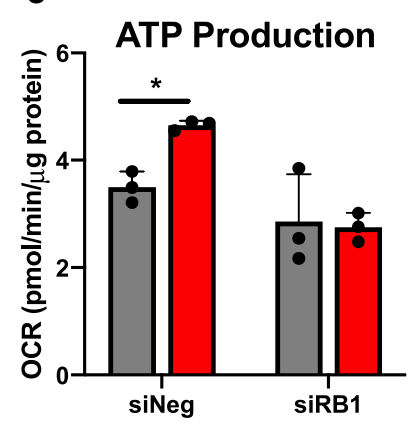

C
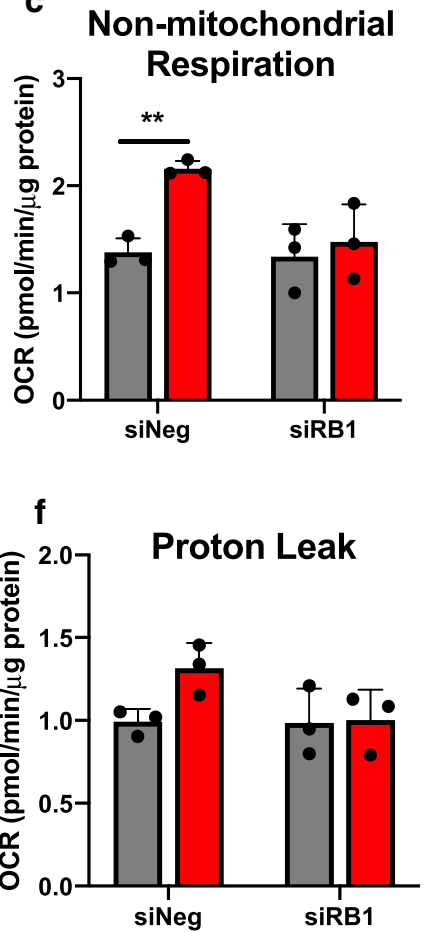

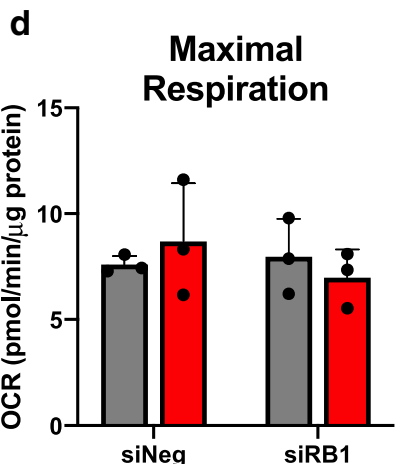

g

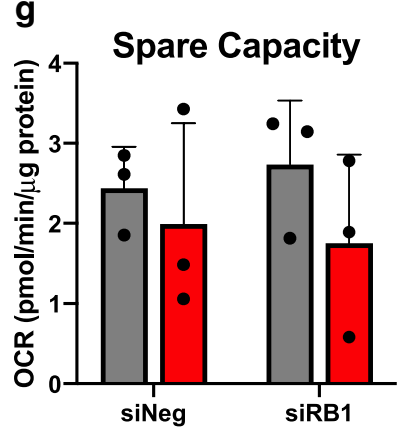

\section{Vehicle}

Palbociclib

Fig. 4 Palbociclib increases mitochondrial respiration in A549 cells. a Oxygen consumption rate (OCR) measured in A549 cells following knockdown of RB and 48-h treatment with $1 \mu \mathrm{M}$ palbociclib. $\mathbf{b}-\mathbf{g}$ OCR assessment of $\mathbf{b}$ basal respiration, $\mathbf{c}$ non-mitochondrial respiration, $\mathbf{d}$ maximal respiration, e ATP production, $\mathbf{f}$ proton leak, and $\mathbf{g}$ spare capacity. Values represent mean $\pm S D$, analyzed by two-way ANOVA $(n=3$, independent experiments). Statistical significances between vehicle and palbociclib treatment are as follows: ${ }^{*} p=0.034,{ }^{* *} p=0.01$, and ${ }^{* * *} p=0.002$

glucose and glutamine utilization. Specifically, palbociclib decreases glucose metabolism through the PPP via inhibition of G6PD activity (Fig. 3a-c), while increasing glutaminolysis to maintain basal mitochondrial function (Fig. 6c, f). Moreover, both changes observed were rescued upon knockdown of $R B$, suggesting the metabolic consequences of CDK4/6 inhibition in A549 cells are RB-dependent.

Recently, it has been demonstrated that CDKs can directly regulate metabolism via phosphorylation of metabolic enzymes. Specifically, the cyclin D3/CDK6 kinase complex can phosphorylate pyruvate kinase M2 


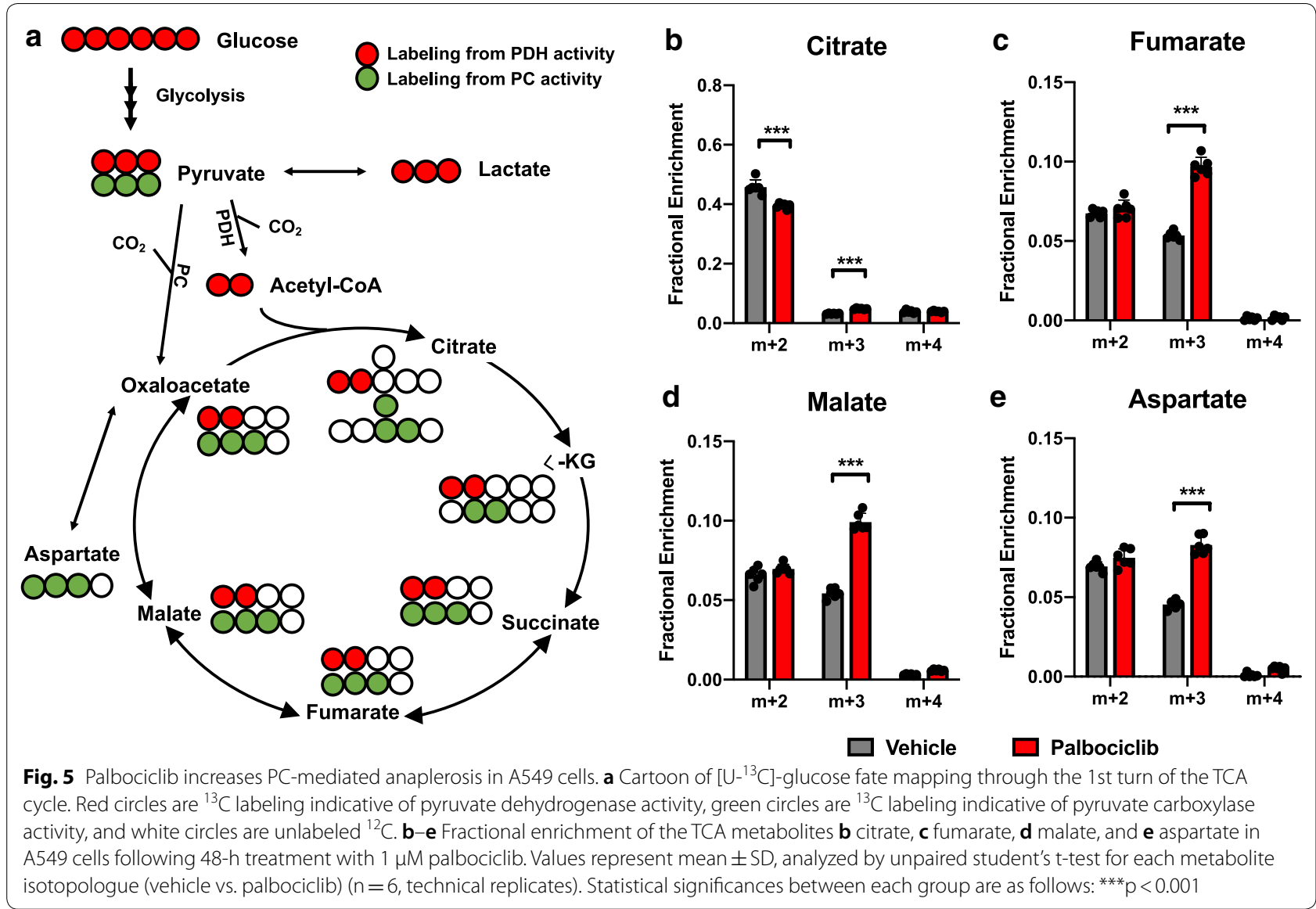

and 6-phosphofructokinase [16]. The authors report this results in shunting of glycolytic intermediates into the PPP and the serine synthesis pathways, and treatment with palbociclib reduces this flux, resulting in NADPH and glutathione depletion and inducing apoptosis. We also observed a decrease in glucose carbon incorporation into nucleotides (Fig. 3a). This was in part due to decreased activity of the rate limiting enzyme in the PPP, G6PD (Fig. 3c). As A549 cells express both CDK4 and CDK6, it remains unclear whether the observed decrease in G6PD activity is due to the inhibitory effects of the cyclin D3/CDK6 complex.

As previously described, palbociclib increases mitochondrial activity in vitro $[14,15]$. Specifically, CDK4/6 inhibition increases glutamine utilization and sensitivity to glutaminase inhibition in breast and colorectal cancer cells [15]. The authors reported that the increase in glutaminolysis was a result of enhanced MYC signaling in response to CDK4/6 inhibition. We did observe a modest increase in basal oxygen consumption upon palbociclib treatment (Fig. 4b), which is driven, in part, by enhanced glutaminolysis (Fig. 6c, f). This sensitized A549 cells to treatment with CB-839 (Fig. 6h). MYC exerts its effect on glutaminolysis in part via upregulation of glutaminase $[39,40]$. As we did not observe a significant increase in GLS expression (Fig. 6g), our data suggest the enhanced glutaminolysis in this context may be independent of MYC signaling.

While other studies have reported enhanced glycolysis upon CDK4/6 inhibition or deletion, or direct activation of RB itself $[14,15,41]$, our $\left[\mathrm{U}_{-}{ }^{13} \mathrm{C}\right]$-glucose and ECAR studies revealed no significant changes in glycolytic function upon palbociclib treatment (Figs. 1, 2). One possible explanation for this discrepancy is the more acute treatment duration for our studies (48-h compared to 5-7 days). To address this, cells were treated with palbociclib for 5-days and, similar to 48-h exposure, we did not observe any differences in glycolytic parameters within the glycolysis stress test (Additional file 1: Figure S3). Prolonged CDK4/6 inhibition ( $96 \mathrm{~h}$ to 2 weeks) has been reported to induce both senescence and autophagy in vitro [14, $15,42,43]$. Many of the reported metabolic consequences of longer-term palbociclib treatment are also metabolic hallmarks of these cellular processes. Senescent cells exhibit elevated glycolysis and mitochondrial 


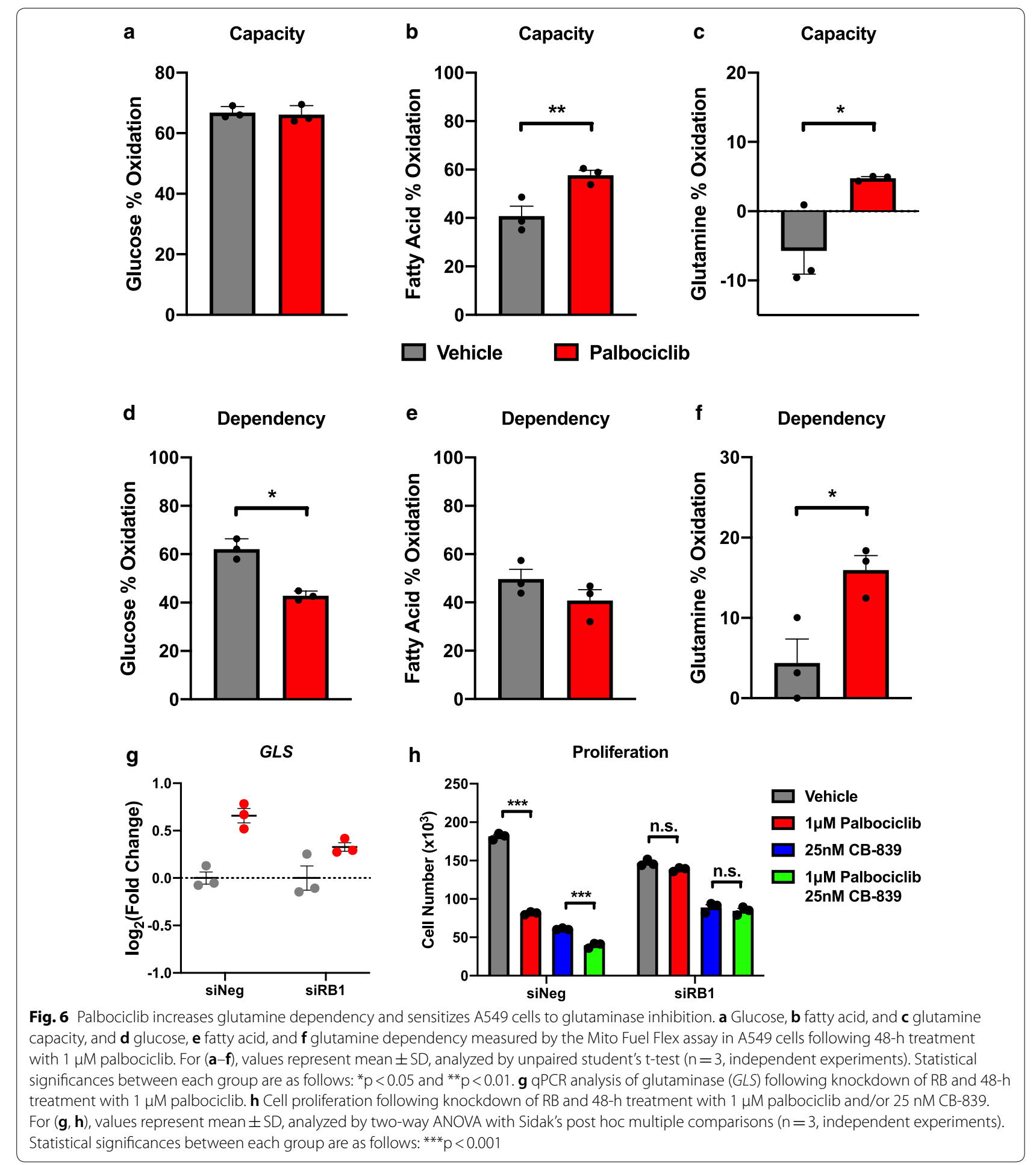

metabolism, resulting in increased mitochondrial mass and production of reactive oxygen species (ROS) [44, 45]. This triggers autophagy and protein degradation to mitigate the cellular stress induced by senescence [46]. Recently, palbociclib-mediated induction of these cellular responses has been found to be dependent on both RB expression and lack of low molecular weight cyclin E (LMWE) [43]. As A549 cells express LMWE, this cell model is presumed to be resistant to senescence or autophagy upon palbociclib treatment [43]. 
This may explain the lack of glycolytic changes in A549 cells under prolonged exposure compared to other cell types $[14,15]$ and may need to be considered when assessing metabolic responses to palbociclib across different cancer models. Our studies are unique in that we have identified a metabolic response in A549 cells under shorter-term CDK4/6 inhibition.

Given the recent use of palbociclib in clinical trials for NSCLC patients, understanding the functional consequences of CDK4/6 inhibition on cancer cell metabolism are important for identifying potential combination therapies to improve patient outcome. Our studies in A549 cells demonstrate that palbociclib treatment increases glutamine dependency and sensitizes cells to the glutaminase inhibitor, CB-839. While the precise mechanism by which palbociclib mediates increased glutaminolysis remains unclear, RB expression is required for palbociclib to exert its function in this context. A noted limitation to this work is that the analysis on the metabolic response to palbociclib is solely performed within the A549 cell line. Further work will not only be necessary to define the cellular mechanisms for these palbociclib effects but also required in additional models to more firmly establish these metabolic changes in response to drug treatment across NSCLC in general. Our data highlight the metabolic changes upon palbociclib treatment in A549 cells and may support the of targeting CDK4/6 inhibition in combination with glutaminase inhibitors in NSCLC patients with RB-proficient tumors.

\section{Conclusions}

Herein, we report that palbociclib treatments results in a metabolic shift in A549 cells. Specifically, we found that palbociclib decreases glucose metabolism through the PPP via inhibition of G6PD activity, while increasing dependency on glutaminolysis to maintain basal mitochondrial function While the precise mechanism by which palbociclib mediates its metabolic functions remain unclear, its effect on glucose and glutamine utilization in A549 cells are RB-dependent. Together, our data define the metabolic impact of CDK4/6 inhibition in a NSCLC cell type and highlight unique vulnerabilities that may be targeted using combination therapy with palbociclib treatment.

\section{Supplementary information}

Supplementary information accompanies this paper at https://doi. org/10.1186/s12935-020-01357-x.

Additional file 1: Figure S1. Palbociclib decreases RB phosphorylation and proliferation of A549 lung cancer cells. Figure S2. Confirmation of siRNA-mediated knock-down of RB in A549 cells. Figure S3. Longer exposure to palbociclib does not alter glycolytic function in A549 cells.

\section{Abbreviations}

NSCLC: Non-small cell lung cancer; RB: Retinoblastoma protein; CDK4/6: Cyclin-dependent kinase 4/6; PPP: Pentose phosphate pathway; ECAR: Extracellular acidification rate; G6PD: Glucose 6-phosphate dehydrogenase; PRPS2: Phosphoribosyl pyrophosphate synthetase 2; RRM1/2: Ribonucleotide reductase 1/2; OCR: Oxygen consumption rate; $\mathrm{PDH}$ : Pyruvate dehydrogenase; PC: Pyruvate carboxylase; GLS: Glutaminase; ROS: Reactive oxygen species.

\section{Acknowledgements}

We would like to thank Dr. Bradford Hill and Lindsey McNally for their assistance with the Seahorse XFe96 Analyzer studies.

\section{Authors' contributions}

LRC and BFC designed the study. LRC performed all of the studies and LH and $X Y$ contributed to the mass spectrometry experiments. LRC and PL analyzed the metabolomics data and SNR assisted in the statistical analysis. LRC and BFC prepared the manuscript and XZ and BFC contributed funding for the project. All authors read and approved the final manuscript.

\section{Funding}

This work was supported by NIH Grants S10OD020106 (XZ) and R01CA166327 (BFC) and American Cancer Society, RSG 13-139-01-CNE (BFC).

Availability of data and materials

Not applicable.

Ethics approval and consent to participate

Not applicable.

\section{Consent for publication}

Not applicable.

\section{Competing interests}

The authors declare that they have no competing interests.

\section{Author details}

1 Department of Biochemistry and Molecular Genetics, University of Louisville, Louisville, KY, USA. ${ }^{2}$ Diabetes and Obesity Center, Christina Lee Brown Envirome Institute, Louisville, KY, USA. ${ }^{3}$ Department of Chemistry, Center for Regulatory and Environmental Analytical Metabolomics, University of Louisville, Louisville, KY, USA. ${ }^{4}$ Department of Bioinformatics and Biostatistics, University of Louisville, Louisville, KY, USA. ${ }^{5}$ Biostatistics and Bioinformatics Facility, James Graham Brown Cancer Center, University of Louisville, Louisville, KY, USA.

${ }^{6}$ James Graham Brown Cancer Center, Louisville, KY, USA. ${ }^{7}$ Present Address:

Department of Neuroscience, University of Kentucky, Lexington, KY, USA.

Received: 10 April 2020 Accepted: 16 June 2020

Published online: 01 July 2020

\section{References}

1. Zappa C, Mousa SA. Non-small cell lung cancer: current treatment and future advances. Transl Lung Cancer Res. 2016;5(3):288-300.

2. Gridelli C, Rossi A, Carbone DP, Guarize J, Karachaliou N, MokT, et al. Nonsmall-cell lung cancer. Nat Rev Dis Primers. 2015;1:15009.

3. Torre LA, Bray F, Siegel RL, Ferlay J, Lortet-Tieulent J, Jemal A. Global cancer statistics, 2012. CA Cancer J Clin. 2015;65(2):87-108.

4. Esposito V, Baldi A, Tonini G, Vincenzi B, Santini M, Ambrogi V, Mineo TC, Persichetti P, Liuzzi G, Montesarchio V, Wolner E, Baldi F, Groeger AM. Analysis of cell cycle regulator proteins in non-small cell lung cancer. J Clin Pathol. 2004;57:58-63.

5. Eymin B, Gazzeri S. Role of cell cycle regulators in lung carcinogenesis. Cell Adhes Migrat. 2009;4:114-23.

6. Goel S, DeCristo MJ, McAllister SS, Zhao JJ. CDK4/6 inhibition in cancer: beyond cell cycle arrest. Trends Cell Biol. 2018;28(11):911-25.

7. Klein ME, Kovatcheva M, Davis LE, Tap WD, Koff A. CDK4/6 inhibitors: the mechanism of action may not be as simple as once thought. Cancer Cell. 2018;34(1):9-20.

8. Beaver JA, Amiri-Kordestani L, Charlab R, Chen W, Palmby T, Tilley A, et al. FDA approval: palbociclib for the treatment of postmenopausal patients 
with estrogen receptor-positive, HER2-negative metastatic breast cancer. Clin Cancer Res. 2015;21(21):4760-6.

9. Gopalan PK, Villegas AG, Cao C, Pinder-Schenck M, Chiappori A, Hou W, et al. CDK4/6 inhibition stabilizes disease in patients with p16-null nonsmall cell lung cancer and is synergistic with MTOR inhibition. Oncotarget. 2018;9(100):37352-66.

10. Liu M, Xu S, Wang Y, Li Y, Li Y, Zhang H, et al. PD 0332991, a selective cyclin D kinase $4 / 6$ inhibitor, sensitizes lung cancer cells to treatment with epidermal growth factor receptor tyrosine kinase inhibitors. Oncotarget. 2016;7(51):84951-64.

11. Nie H, Zhou X, Shuzhang D, Nie C, Zhang X, Huang J. Palbociclib overcomes afatinib resistance in non-small cell lung cancer. Biomed Pharmacother. 2019;109:1750-7.

12. Pacheco J, Schenk E. CDK4/6 inhibition alone and in combination for non-small cell lung cancer. Oncotarget. 2019;10(6):618-9.

13. Zhou J, Zhang S, Chen X, Zheng X, Yao Y, Lu G, et al. Palbociclib, a selective CDK4/6 inhibitor, enhances the effect of selumetinib in RAS-driven non-small cell lung cancer. Cancer Lett. 2017;408:130-7.

14. Franco J, Balaji U, Freinkman E, Witkiewicz AK, Knudsen ES. Metabolic reprogramming of pancreatic cancer mediated by CDK4/6 inhibition elicits unique vulnerabilities. Cell Rep. 2016;14(5):979-90.

15. Tarrado-Castellarnau M, de Atauri P, Tarrago-Celada J, Perarnau J, Yuneva M, Thomson TM, et al. De novo MYC addiction as an adaptive response of cancer cells to CDK4/6 inhibition. Mol Syst Biol. 2017;13(10):940.

16. Wang H, Nicolay BN, Chick JM, Gao X, Geng Y, Ren H, et al. The metabolic function of cyclin D3-CDK6 kinase in cancer cell survival. Nature. 2017:546(7658):426-30.

17. Klavins K, Drexler H, Hann S, Koellensperger G. Quantitative metabolite profiling utilizing parallel column analysis for simultaneous reversed-phase and hydrophilic interaction liquid chromatography separations combined with tandem mass spectrometry. Anal Chem. 2014;86(9):4145-50.

18. Adusumilli R, Mallick P. Data conversion with ProteoWizard msConvert Methods Mol Biol. 2017;1550:339-68.

19. Agrawal S, Kumar S, Sehgal R, George S, Gupta R, Poddar S, et al. ElMAVEN: a fast, robust, and user-friendly mass spectrometry data processing engine for metabolomics. In: D'Alessandro A, editor. High-throughput metabolomics: methods and protocols. 1978th ed. New York: Springer; 2019

20. Salabei JK, Lorkiewicz PK, Mehra P, Gibb AA, Haberzettl P, Hong KU, et al. Type 2 diabetes dysregulates glucose metabolism in cardiac progenitor Cells. J Biol Chem. 2016:291(26):13634-48.

21. Wei X, Lorkiewicz PK, Shi B, Salabei JK, Hill BG, Kim S, et al. Analysis of stable isotope assisted metabolomics data acquired by high resolution mass spectrometry. Anal Methods. 2017;9:2275-83.

22. Lorkiewicz PK, Gibb AA, Rood BR, He L, Zheng Y, Clem BF, et al. Integration of flux measurements and pharmacological controls to optimize stable isotope-resolved metabolomics workflows and interpretation. Scientific Reports. 2019;9(1):1-7.

23. Cretella D, Fumarola C, Bonelli M, Alfieri R, La Monica S, Digiacomo G, et al. Pre-treatment with the CDK4/6 inhibitor palbociclib improves the efficacy of paclitaxel in TNBC cells. Sci Rep. 2019;9(1):13014.

24. Cretella D, Ravelli A, Fumarola C, La Monica S, Digiacomo G, Cavazzoni $A$, et al. The anti-tumor efficacy of CDK4/6 inhibition is enhanced by the combination with PI3K/AKT/mTOR inhibitors through impairment of glucose metabolism in TNBC cells. J Exp Clin Cancer Res. 2018;37(1):72.

25. Knudsen ES, Witkiewicz AK. Defining the transcriptional and biological response to CDK4/6 inhibition in relation to ER+/HER2 - breast cancer. Oncotarget. 2016;7(43):69111-23.

26. Daye D, Wellen KE. Metabolic reprogramming in cancer: unraveling the role of glutamine in tumorigenesis. Semin Cell Dev Biol. 2012;23(4):362-9.
27. DeBerardinis RJ, Cheng T. Q's next: the diverse functions of glutamine in metabolism, cell biology and cancer. Oncogene. 2010;29(3):313-24.

28. DeBerardinis RJ, Mancuso A, Daikhin E, Nissim I, Yudkoff M, Wehrli S, et al. Beyond aerobic glycolysis: transformed cells can engage in glutamine metabolism that exceeds the requirement for protein and nucleotide synthesis. Proc Natl Acad Sci USA. 2007;104(49):19345-50.

29. Boysen G, Jamshidi-Parsian A, Davis MA, Siegel ER, Simecka CM, Kore RA, et al. Glutaminase inhibitor CB-839 increases radiation sensitivity of lung tumor cells and human lung tumor xenografts in mice. Int J Radiat Biol. 2019;95(4):436-42.

30. Cairns RA, Harris IS, MakTW. Regulation of cancer cell metabolism. Nat Rev Cancer. 2011;11(2):85-95.

31. DeBerardinis RJ, Chandel NS. Fundamentals of cancer metabolism. Sci Adv. 2016;2(5):e1600200.

32. DeBerardinis RJ, Lum JJ, Hatzivassiliou G, Thompson CB. The biology of cancer: metabolic reprogramming fuels cell growth and proliferation. Cell Metab. 2008;7(1):11-20

33. Martinez-Outschoorn UE, Peiris-Pages M, Pestell RG, Sotgia F, Lisanti MP. Cancer metabolism: a therapeutic perspective. Nat Rev Clin Oncol. 2017;14(1):11-31

34. Pavlova NN, Thompson CB. The emerging hallmarks of cancer metabolism. Cell Metab. 2016;23(1):27-47.

35. Blanchet E, Annicotte JS, Lagarrigue S, Aguilar V, Clape C, Chavey C, et al. E2F transcription factor-1 regulates oxidative metabolism. Nat Cell Biol. 2011;13(9):1146-52.

36. Bonelli M, La Monica S, Fumarola C, Alfieri R. Multiple effects of CDK4/6 inhibition in cancer: from cell cycle arrest to immunomodulation. Biochem Pharmacol. 2019;170:113676.

37. Burkhart DL, Sage J. Cellular mechanisms of tumour suppression by the retinoblastoma gene. Nat Rev Cancer. 2008;8(9):671-82.

38. Clem BF, Chesney J. Molecular pathways: regulation of metabolism by RB. Clin Cancer Res. 2012;18(22):6096-100.

39. Gao P, Tchernyshyov I, Chang TC, Lee YS, Kita K, Ochi T, et al. c-Myc suppression of miR-23a/b enhances mitochondrial glutaminase expression and glutamine metabolism. Nature. 2009;458(7239):762-5.

40. Goetzman ES, Prochownik EV. The role for Myc in coordinating glycolysis, oxidative phosphorylation, glutaminolysis, and fatty acid metabolism in normal and neoplastic tissues. Front Endocrinol. 2018:9:129.

41. Takebayashi S, Tanaka H, Hino S, Nakatsu Y, Igata T, Sakamoto A, et al. Retinoblastoma protein promotes oxidative phosphorylation through upregulation of glycolytic genes in oncogene-induced senescent cells. Aging Cell. 2015;14(4):689-97.

42. Thangavel C, Boopathi E, Liu Y, McNair C, Haber A, Perepelyuk M, et al. Therapeutic challenge with a CDK 4/6 inhibitor induces an RB-dependent SMAC-mediated apoptotic response in non-small cell lung cancer. Clin Cancer Res. 2018;24:1402-14.

43. Vijayaraghavan S, Karakas C, Doostan I, Chen X, Bui T, Yi M, et al. CDK4/6 and autophagy inhibitors synergistically induce senescence in $\mathrm{Rb}$ positive cytoplasmic cyclin E negative cancers. Nat Commun. 2017;8:15916.

44. Kwon SM, Hong SM, Lee YK, Min S, Yoon G. Metabolic features and regulation in cell senescence. BMB Rep. 2019;52(1):5-12.

45. Salama R, Sadaie M, Hoare M, Narita M. Cellular senescence and its effector programs. Genes Dev. 2014;28(2):99-114.

46. Rabinowitz JD, White E. Autophagy and metabolism. Science. 2010:330(6009):1344-8.

\section{Publisher's Note}

Springer Nature remains neutral with regard to jurisdictional claims in published maps and institutional affiliations. 